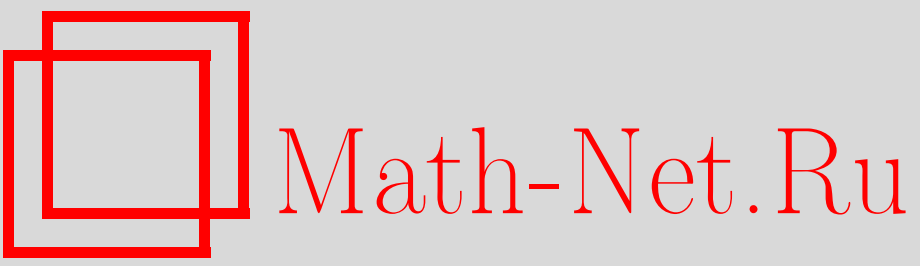

А. Д. Миронов, Самодуальные гамильтонианы как деформации свободных систем, ТМФ, 2001, том 129, номер 2, 327-332

DOI: https://doi.org/10.4213/tmf539

Использование Общероссийского математического портала Math-Net.Ru подразумевает, что вы прочитали и согласны с пользовательским соглашением

http://www.mathnet.ru/rus/agreement

Параметры загрузки:

IP : 34.229 .108 .108

26 апреля 2023 г., 13:06:07 
ТЕОРЕТИЧЕСКАЯ

И МАТЕМАТИЧЕСКАЯ

ФИЗИКА

Том 129, № 2

ноябрь, 2001

(C) 2001 г.

А. Д. Миронов*

\section{САМОДУАЛЬНЫЕ ГАМИЛЬТОНИАНЫ КАК ДЕФОРМАЦИИ СВОБОДНЫХ СИСТЕМ}

Сформулирована проблема нахождения самодуальных гамильтонианов (ассоциированных с интегрируемыми системами) как деформаций свободных систем, заданных на различных симплектических многообразиях. Обсуждаются несколько явных известных примеров, включая обнаруженные недавно дваждыэллиптические гамильтонианы. Как основное рассматривается именно понятие самодуальности, в то время как дуальность в интегрируемых системах (семейства Тоды, Калоджеро, Руджинарса) оказывается производным понятием (вырождением самодуальных систем).

1. Новый всплеск интереса к теории интегрируемых многочастичных систем, происходящий в течение последних нескольких лет, связан в основном с неожиданным открытием взаимосвязи между $N=2$ суперсимметричными калибровочными теориями [1] и интегрируемыми системами [2] (см. также [3] и ссылки там). В действительности исследования интегрируемых систем все еще получают новую мотивацию из физики. $\mathrm{K}$ примеру, необходимость описания шестимерных калибровочных теорий привела к понятию дваждыэллиптических систем [4], [5]. Эти системы были сконструированы с использованием понятия дуальности [4], которое возникло в физической теории [6].

Дуальность в интегрируемых системах впервые наблюдалась Руджинарсом [7] в многочастичных рациональных и тригонометрических системах Калоджеро и Руджинарса. С тех пор дуальность была обобшена на эллиптические системы [4], [5], [8]. Однако само это понятие до сих пор не было однозначно определено. Более того, похоже, что сушествует великое множество дуальных систем, так что их нахождение не представляет ни малейшей проблемы!

В настоящей работе мы обсуждаем возможность начать с более ограничительного понятия самодуальности. Оно может быть легче формализовано, естественно приводит к понятию дуальности и, являясь весьма ограничительным, немедленно приводит к задаче построения новых/всех самодуальных систем. Здесь мы просто ограничиваемся несколькими явными примерами самодуальных систем. Мы также не обсуждаем симплектическую геометрию, стоящую за понятием самодуальности.

\footnotetext{
* Физический институт им. П.Н. Лебедева РАН, Институт теоретической и экспериментальной физики, Москва, Россия. E-mail: mironov@lpi.ac.ru, mironov@itep.ru
} 
Стандартные факты и известные примеры дуальных систем, как и подходящая техника работы с ними, могут быть найдены в обзорах [9].

2. Рассмотрим свободную гамильтонову систему с $N$ степенями свободы, фазовым пространством (симплектическим многообразием) которой является $\mathbb{R}^{2 N}$. В координатах Дарбу симплектическая форма равна $\sum_{i} d p_{i} \wedge d q_{i}$. В этом случае существует $N$ независимых функций на фазовом пространстве - интегралов движения, находяшихся в инволюции (не важно, какой из этих интегралов выбран в качестве гамильтониана). Выбор этих интегралов ничем не ограничен, например это могут быть просто симметрические степени $p_{i}: H_{k}(p, q)=\sum_{i} p_{i}^{k}$.

Не сушествует большего числа независимых интегралов, находяшихся в инволюции, скажем некоторых функций координат. Поэтому сделанный выбор в некотором смысле нарушает симметрию относительно перестановок $\{p\}$ и $\{q\}$ (т.е. фиксирует поляризацию). Для того чтобы восстановить эту симметрию, нужно проделать (анти)каноническое преобразование $p_{i} \rightarrow Q_{i}, q_{i} \rightarrow P_{i}$. В новых координатах $\widetilde{H}_{k}(P, Q)=H_{k}(p, q)=$ $\sum_{i} Q_{i}^{k}$, в то время как $\sum_{i} q_{i}^{k}=H_{k}(P, Q)$. Здесь $\widetilde{H}_{k}(P, Q)$ - интегралы (гамильтонианы), полученные в результате канонического преобразования.

Все это означает, что действие симплектической группы на гамильтонианах должно сопровождаться подходящей (канонической) заменой переменных на симплектическом многообразии (что-то вроде свойства ковариантности). Это можно также описать парой соотношений

$$
H_{k}(p, q)=\sum_{i} Q_{i}^{k}, \quad H_{k}(P, Q)=\sum_{i} q_{i}^{k}
$$

Эти соотношения явно реализуют симметрию между переменными $\{Q\}$ (по сути, старые $\{p\}$ и $\{q\})$.

Рассмотрим теперь менее тривиальную взаимодействующую систему. Мы потребуем, чтобы она была интегрируема в смысле Лиувилля, т.е. должно существовать $N$ независимых интегралов движения в инволюции. В этом случае гамильтонианы могут быть весьма сложными функциями на фазовом пространстве. Можно задаться вопросом, как обобшить симметрию между координатами и импульсами на этот случай. Для того чтобы это сделать, можно просто использовать те же самые соотношения (1).

Это означает, что во взаимодействующей системе на первом этапе нужно сделать каноническое преобразование к свободной системе (по сути, к переменным действия), а затем проделать антиканоническое преобразование, которое просто переставляет импульсы и координаты. Заметим, что соотношения (1) уже содержат $2 N$ уравнений, и имеется еще дополнительно условие каноничности преобразования $p_{i} \rightarrow Q_{i}, q_{i} \rightarrow P_{i}$. Это ограничивает гамильтонианы.

Рассмотрим теперь более общую ситуацию, когда фазовое пространство не плоское и может быть компактным. Тогда даже свободная система может иметь менее тривиальные гамильтонианы $h_{k}(p)$, так как они должны быть “хорошими” функциями на фазовом пространстве. В этом случае уравнения (1) принимают вид

$$
H_{k}(p, q)=h_{k}(Q), \quad H_{k}(P, Q)=h_{k}(q)
$$


ОПреДЕЛЕНИЕ. Система с гамильтонианами, которые удовлетворяют уравнениям $(2)$ с переменными $(p, q)$ и $(P, Q)$, связанными каноническим преобразованием, называется самодуальной.

Нам неизвестен конструктивный путь построения таких систем, поэтому мы ограничимся несколькими явными примерами.

Заметим, что соотношения (2) обладают свойством, которое можно было бы назвать ковариантностью: вместо набора свободных гамильтонианов $h_{k}$ можно использовать какой-нибудь другой набор подходяших функций на фазовом пространстве. Все они являются некоторыми функциями первоначальных $h_{k}$, а новые дуальные гамильтонианы такими же функциями старых дуальных гамильтонианов.

3. Мы будем рассматривать комплексифицированную версию фазового пространства, так что гамильтонианы должны обладать хорошими аналитическими свойствами на фазовом пространстве. Однако, как правило, фазовое пространство сильно асимметрично относительно изменения поляризации, т.е. перестановки импульсов и координат. В этом случае невозможно пользоваться формулами, подобными (2).

Нашей стратегией в таких случаях будет конструирование самодуальных систем на нетривиальном фазовом пространстве, которое зависит от модулей, а затем переход к границе пространства модулей. Это приводит к различным вырождениям, так что, например, только координаты $q$, но не $Q$, попадают в вырожденную область. В окрестности границы два соотношения в (2) выглядят по-разному:

$$
H_{k}^{(1)}(p, q)=h_{k}^{(1)}(Q), \quad H_{k}^{(2)}(P, Q)=h_{k}^{(2)}(q),
$$

и задают два различных набора гамильтонианов. Мы называем эти гамильтонианы дуальнылми. Дуальные гамильтонианы - это просто гамильтонианы самодуальной системы в двух различны $x$ областях пространства модулей. Иными словами, это означает, что нужно использовать те же два уравнения (2), но переставить в них точки пространства модулей:

$$
H_{k}^{(1,2)}(p, q)=H_{k}\left(p, q ; m_{1,2}\right), \quad h_{k}^{(1,2)}(q)=h_{k}\left(q ; m_{1,2}\right)
$$

Следовательно, можно получить аналог самодуальности для более сложных фазовых пространств при помощи вырождения. В действительности обратное также верно: дуальная система оказалась мощным техническим средством при конструировании самодуальных гамильтонианов, которые можно строить, начиная с дуальных гамильтонианов. Построение последних часто проще.

Начнем с системы с одной степенью свободы (она также описывает системы с двумя степенями свободы с отщепляющимся центром масс). В этом случае новые переменные $P$ и $Q$ даются двумя соотношениями $(2)$, и нужно еше наложить условие каноничности преобразования

$$
\frac{\partial H(p, q)}{\partial p} h^{\prime}(q)=\frac{\partial H(P, Q)}{P} h^{\prime}(Q) .
$$


Снова используя соотношения (2), можно переписать правую часть условия (4) как функцию $p$ и $q$ и получить сложное функциональное дифференциальное уравнение, которое определяет гамильтониан самодуальной системы. Конечно, мы не можем решить это уравнение и даже сказать, сколько у него решений. Однако это одно уравнение на одну функцию.

В системе с $N$ степенями свободы соотношения (2) опять определяют новые координаты и импульсы, в то время как условие каноничности задает систему уравнений на гамильтониан.

4. Перейдем теперь к конкретным примерам. Начнем с простейшего случая систем с одной степенью свободы. Свободные гамильтонианы мы выбираем в форме, приводящей к некоторым стандартным гамильтоновым системам.

ПримеР 1. Фазовое пространство $\mathcal{M}=\mathbb{R}^{2}$. Свободная система дается гамильтонианом $H=p^{2} / 2$. Решением уравнений самодуальности (1) является функция $H=p^{2} / 2+$ $g^{2} / q^{2}$, где $g$ - произвольньй параметр. Это гамильтониан рациональной системы Калоджеро с константой связи $g$.

Пример 2. Фазовое пространство $\mathcal{M}=S^{1} \times S^{1}$, произведение двух окружностей с радиусами $R_{1}$ и $R_{2}$. Свободная система дается гамильтонианом $H=$ $\cos \left(p / R_{1}\right)$. Самодуальный гамильтониан - гамильтониан системы Руджинарса $H=$ $\sqrt{1-2 g^{2} / \sin ^{2}\left(q / R_{2}\right)} \cos \left(p / R_{1}\right)$. Радиусы окружностей задают пространство модулей теории. Условия самодуальности имеют вид

$$
H\left(p, q ; R_{1}, R_{2}\right)=\cos \left(\frac{Q}{R_{1}}\right), \quad H\left(p, q ; R_{2}, R_{1}\right)=\cos \left(\frac{q}{R_{2}}\right) .
$$

При вырождении импульсной окружности $\left(R_{1} \rightarrow \infty\right)$ можно получить гамильтониан тригонометрической системы Калоджеро $H_{\mathrm{tC}}$, а при вырождении координатной - гамильтониан рациональной системы Руджинарса $H_{\mathrm{rR}}$, так что соотношения (5) становятся соотношениями дуальности:

$$
\begin{aligned}
& \left.H\left(p, q ; R_{1}, R_{2}\right)\right|_{R_{1}=\infty} \equiv H_{\mathrm{tC}}(p, q)=\frac{Q^{2}}{2} \\
& \left.H\left(p, q ; R_{2}, R_{1}\right)\right|_{R_{1}=\infty} \equiv H_{\mathrm{rR}}(P, Q)=\cos \left(\frac{q}{R_{2}}\right) .
\end{aligned}
$$

ПРимеР 3. Можно рассмотреть комплексифицированное фазовое пространство из примера 2, т.е. координату и импульс, заданные на цилиндре. Тогда имеется еше одно решение уравнений самодуальности, в котором тригонометрические функции заменены гиперболическими.

ПримеР 4. Обобщая пример 3, можно рассмотреть координату и импульс, заданные на торе. Свободная система дается гамильтонианом $H=\operatorname{cn}(p \mid k)$, эллиптическим косинусом (Якоби) с эллиптическим модулем $k$. Для того чтобы решить уравнения самодуальности (2) в этом случае, можно сначала решить их в вырожденном 
случае, например когда импульсный тор вырождается в сферу и гамильтониан $h^{(1)}(Q)$ в (3) равен $Q^{2} / 2$ (в то время как $h^{(2)}(q)=\operatorname{cn}(q \mid k)$ ). Тогда естественно предположить, что $H^{(1)}(p, q)$ - эллиптический гамильтониан Калоджеро-Мозера, $H(p, q)=p^{2} / 2+$ $g^{2} / \operatorname{sn}^{2}(q \mid k)$, и получить, решая уравнения (3),

$$
\begin{gathered}
H(P, Q)=\operatorname{cn}(q \mid k)=\alpha(Q) \operatorname{cn}\left(P \sqrt{k^{\prime 2}+k^{2} \alpha^{2}(Q)} \mid \frac{k \alpha(Q)}{\sqrt{k^{\prime 2}+k^{2} \alpha^{2}(Q)}}\right), \\
\alpha^{2}(Q)=1-\frac{2 g^{2}}{Q^{2}}, \quad k^{\prime 2} \equiv 1-k^{2} .
\end{gathered}
$$

Этот результат подсказьвает анзац для самодуального случая [4]:

$$
H(P, Q \mid k, \tilde{k})=\alpha(Q \mid k, \tilde{k}) \operatorname{cn}(P \beta(Q \mid k, \tilde{k}) \mid \gamma(Q \mid k, \tilde{k})) .
$$

Подставляя его в уравнения самодуальности (2) (причем $k$ и $\tilde{k}$ меняются местами), можно получить

$$
\begin{gathered}
\alpha^{2}(q \mid \tilde{k}, k)=1-\frac{2 \nu^{2}}{\operatorname{sn}^{2}(q \mid k)} \\
\beta^{2}(q \mid \tilde{k}, k)=\tilde{k}^{\prime 2}+\tilde{k}^{2} \alpha^{2}(q \mid k), \quad \gamma^{2}(q \mid \tilde{k}, k)=\frac{\tilde{k}^{2} \alpha^{2}(q \mid k)}{\tilde{k}^{\prime 2}+\tilde{k}^{2} \alpha^{2}(q \mid k)} .
\end{gathered}
$$

Эта система называется дваждыэллиптической системой [4] (подробнее см. [9]).

Подобным образом можно найти многочастичные обобщения. Техника для изучения таких систем была развита в работах [4], [7]-[10]. Однако до сих пор найденные решения - это только многочастичные рациональные системы Калоджеро, тригонометрические системы Руджинарса и многочастичная дваждыэллиптическая система, построенная не вполне явно.

5. Итак, мы ввели понятие самодуальности (дуальности) и построили несколько явных примеров. Мы обсуждали только классические гамильтоновы системы, хотя существует совершенно прямое обобшение на квантовый случай [7], [5]. Действительно, нужно просто рассмотреть пару наборов уравнений Шредингера

$$
\widehat{H}_{k}\left(\partial_{x}, x\right) \Psi(x ; \lambda)=h_{k}(\lambda) \Psi(x ; \lambda), \quad \widehat{H}_{k}\left(\partial_{\lambda}, \lambda\right) \Psi(x ; \lambda)=h_{k}(x) \Psi(x ; \lambda),
$$

где переменная $\lambda$ играет роль (функции) энергии в первом наборе уравнений, а переменная $x-$ во втором.

Имеется также еше два вопроса, оставшихся за рамками данной статьи. Прежде всего, отметим, что понятие дуальности можно обобщить на деформации несвободных систем. С этой целью нужно просто допускать в правых частях уравнений (2) произвольные гамильтонианы.

Другая важная не обсуждавшаяся здесь проблема - геометрический смысл самодуальности. Поскольку самодуальность обеспечивает симметрию между старыми $(q)$ и новыми $(Q)$ координатами, то следует ожидать явное действие симплектической группы на этих координатах. Это действительно наблюдается в примерах, хотя явная реализация не проста (см. [11]). 
Благодарности. Автор благодарен Г. Брадену, А. Горскому, А. Маршакову, М. Ольшанецкому и Т. Такебе за обсуждения и А. Морозову за ценные обсуждения и чтение текста статьи. Работа была частично поддержана грантами INTAS-00-561, CRDF\#6531, РФФИ № 01-02-17682а.

\section{Список литературы}

[1] N. Seiberg, E. Witten. Nucl. Phys. B. 1994. V. 426. P. 19; V. 431. P. 484.

[2] A. Gorsky, I. Krichever, A. Marshakov, A. Mironov, A. Morozov. Phys. Lett. B. 1995. V. 355. P. 466.

[3] H.W. Braden, I.M. Krichever (Eds.). Integrability: The Seiberg-Witten and Whitham Equations. Amsterdam: Gordon and Breach, 2000.

[4] H. W. Braden, A. Marshakov, A. Mironov, A. Morozov. Nucl. Phys. B. 2000. V. 573. P. 553.

[5] V. Fock, A. Gorsky, N. Nekrasov, V. Rubtsov. JHEP. 2000. V. 0007. P. 028.

[6] A. Gorsky, N. Nekrasov, V. Rubtsov. Hilbert schemes, separated variables, and D-branes. hep-th/9901089.

[7] S. N. Ruijsenaars. Commun. Math. Phys. 1988. V. 115. P. 127.

[8] A. Mironov, A. Morozov. Phys. Lett. B. 2000. V. 475. P. 71; Double elliptic systems: problems and perspectives. hep-th/0001168; A. Gorsky, V. Rubtsov. Dualities in integrable systems: geometric aspects. hep-th/0103004.

[9] A. Gorsky, A. Mironov. Integrable many-body systems and gauge theories. hep-th/0011197; A. Mironov. Seiberg-Witten theory and duality in integrable systems. hep-th/0011093.

[10] O. Babelon, D. Bernard. Phys. Lett. B. 1993. V. 317. P. 363.

[11] V. Fock. Three remarks on group invariants related to flat connections. In: Geometry and Integrable Models. Eds. P. Pyatov, S. Solodukhin. Singapore: World Scientific, 1995. P. 20; $V$. Fock, A. Rosly. Poisson structure on moduli of flat connections on Riemann surfaces and $r$-matrix. math/9802054. 